\title{
Development of a pressure-retained transfer system of seafloor natural gas hydrates
}

\author{
Jia-Wang Chen \\ Associate Professor, Ocean College, Zhejiang University, Zhoushan, \\ People's Republic of China (corresponding author: arwang@zju.edu.cn) \\ (Orcid:0000-0002-6351-0062) \\ Qiao-Ling Gao \\ Student, Ocean College, Zhejiang University, Zhoushan, People's Republic \\ of China

\section{Hou-Hong Liu} \\ Student, Ocean College, Zhejiang University, Zhoushan, People's Republic \\ of China (Orcid:0000-0001-5803-4359)
}

\author{
Han-Quan Zhang \\ Senior Engineer, Guangzhou Marine Geological Survey, Guangzhou, \\ People's Republic of China \\ Bo Xiao \\ Senior Engineer, Guangzhou Marine Geological Survey, Guangzhou, \\ People's Republic of China \\ Fang-Lan Liu \\ Senior Engineer, Guangzhou Marine Geological Survey, Guangzhou, \\ People's Republic of China
}

\begin{abstract}
In order to improve the utilisation rate of the cores of natural gas hydrate samples from the seafloor and broaden the means of the analysis of pressured cores, long-columnar cores obtained by the gravity-piston pressure corer are usually cut into smaller segments, which are then transferred to pressure chambers before they are sent to the laboratory for further analysis. The transfer system for the core of the natural gas hydrate sample in this paper is able to keep the in situ pressure during the transfer process, which can prevent the changing of physicochemical properties of the cores. In addition, the system can also realise the actions of core pushing, cutting and subsampling. The pressure-retaining feature of the transfer system is discussed in this paper, and an experiment was carried out to test the reliability of the pressure retaining of the system. Moreover, the transfer system was tested in the South China Sea twice and showed an excellent performance of pressure maintenance.
\end{abstract}

\author{
Notation \\ $d_{1} \quad$ hole diameter: $\mathrm{mm}$ \\ $d_{0} \quad$ catcher diameter: $\mathrm{mm}$ \\ $L \quad$ catcher length: $\mathrm{mm}$ \\ $P_{1} \quad$ inlet pressure: $\mathrm{MPa}$ \\ $P_{2} \quad$ outlet pressure: $\mathrm{MPa}$ \\ $Q \quad$ flow rate of the medium: $\mathrm{m}^{3} / \mathrm{s}$ \\ $\Delta P \quad$ pressure difference \\ $\delta \quad$ gap width: $\mathrm{m}$ \\ $\mu \quad$ dynamic viscosity of medium \\ $v \quad$ the speed of catcher: $\mathrm{mm} / \mathrm{s}$
}

\section{Introduction}

Natural gas hydrate coring from the seafloor provides samples for the laboratory studying of the physicochemical properties of natural gas hydrates and the condition of mineral reserves, which are of great importance for the development of the mining of seabed resources, as well as environmental protection.

In order to achieve a large sampling scale in the vertical direction for the study of gas hydrates, seabed geology and so on, the cores are required to be long; the longer, the better. For most drilling systems, a single core obtained by a drill is usually longer than $2 \mathrm{~m}$ (Tréhu et al., 2006). For commonly used corers for seafloor sediment, the pressure core barrel (PCB) that is used in the International Deep Sea Drilling Project has a length of $6 \mathrm{~m}$ (Zhu et al., 2011). For the advanced piston corer, which is used in the International Ocean Drilling Program, the sample length can reach $9 \cdot 5 \mathrm{~m}$ (Jutzeler et al., 2014). In China, the long pressure-retained corer developed by groups in Zhejiang University obtained a pressure-retained core with a length of $14.5 \mathrm{~m}$ during the Sea trial in 2011, which is the longest single pressure-retained core in the world (Chen et al., 2013).

However, it is difficult to transport and store these long cores as a whole. Also, to analyse the physicochemical properties of the sample, usually only a small sample from the pressure core is sufficient. Therefore, a pressure core-transfer system with the ability to cut and transfer long cores under pressure is needed (Hao et al., 2013; Rothwell and Rack, 2006). On the other hand, the cores of natural gas hydrate samples need to be stored in a high-pressure environment. The gravity-piston pressure-retained corer can guarantee the high fidelity of deep-sea samples because of its ability to penetrate into seabed sediment; for the precise analysis of the pressure-retained core, the process of cutting and transferring cores should be conducted under in situ pressure (Abegg et al., 2008; Schultheiss et al., 2009). A transfer system for natural gas hydrates usually has the functions of core transferring, core cutting and short columnar core storage. At present, one of the most mature pressure core-transfer systems is the pressure core analysis and transfer system (PCATS) developed by Geotek Company. The maximum operating pressure of the PCATS is $35 \mathrm{MPa}$ with the largest core length of $3.5 \mathrm{~m}$ and the largest core diameter of $63 \mathrm{~mm}$. This system is still widely used in the transfer of natural gas hydrate cores (Priest et al., 2015; Yoneda et al., 2013). Another system used for detecting and analysing natural gas hydrate cores is the pressure core characterisation tools (PCCT) developed by groups in Japan. The core length is $2.6 \mathrm{~m}$, and the maximum operating pressure is $35 \mathrm{MPa}$. The system includes a catcher and a cutting unit 
Environmental Geotechnics

Volume 8 Issue 8
Development of a pressure-retained

transfer system of seafloor natural gas

hydrates

Chen, Gao, Liu et al.
(Santamarina et al., 2015; Yamamoto, 2015; Yoneda et al., 2017), and it can analyse the properties of a sample preliminarily by using the puncturing bit, the acoustic sensor, the shear strength probe and the conductivity probe (Santamarina et al., 2012; Yun et al., 2011).

In order to improve the accuracy of core analysis and realise lowdisturbance transfer at the in situ pressure, the authors developed a ship-borne pressure-retained transfer system transferring the long columnar core of a long gravity-piston pressure corer. The system has the abilities of core gripping, pushing, cutting and subsampling, and, therefore, it is suitable for pressure-retained transfer and pretreatment of cores. The system can handle cores with an outer diameter of $73 \mathrm{~mm}$, and the operating pressure can reach $20 \mathrm{MPa}$ with a change in the inner pressure of less than $20 \%$ during the transfer process of the natural gas hydrate sample. Moreover, the system also considers temperature changes, and the system is put in a temperature-retained container. The types and service conditions of major pressure-retained transfer systems in use are shown in Table 1, including the transfer system presented in this paper.

\section{Components of the system}

The pressure-retained transfer system for the natural gas hydrate core is composed of a pressure-retained corer and a pressureretained transfer device. The pressure-retained corer is used to obtain gas hydrate cores from the seafloor, and the pressureretained transfer device is used to cut and store the original cores. As shown in Figure 1, the pressure-retained transfer device consists of a catcher, a push unit, a cutting unit, ball valves, a sonic detection device and a support device.

Table 1. Current service conditions of major pressure-retained systems

\begin{tabular}{|lccc|}
\hline Parameter & PCATS & PCCT & PTS (this paper) \\
\hline Max. pressure: MPa & 35 & 35 & 20 \\
Temperature: ${ }^{\circ} \mathrm{C}$ & 4 to 30 & - & -2 to 40 \\
Max. length of cores: $\mathrm{m}$ & 3.5 & 1.2 & 1.5 \\
Core diameter: $\mathrm{mm}$ & 63 & - & 67
\end{tabular}

PTS, pressure-retained transfer system

\section{Catcher unit}

The catcher is used for gripping and dragging cores. The length of the stroke of the catcher is around $4 \mathrm{~m}$. In order to realise reciprocating motion, the catcher is fixed on the screw nut, which is driven by a motor, as shown in Figure 2.

In addition, considering the motions of gripping and releasing of the cores, the catcher is designed to be opened and closed by ejector pins. In order to grip cores reliably, the ejector pins, which are specially designed, can be jammed into the core when they are unfolded, as shown in Figure 3. The position of catcher is determined by a counter on the screw and controlled by programming.

\section{Pushing unit}

The pushing unit has a pressure-retained cylinder to accommodate the long core, and it also has a servo motor that controls the

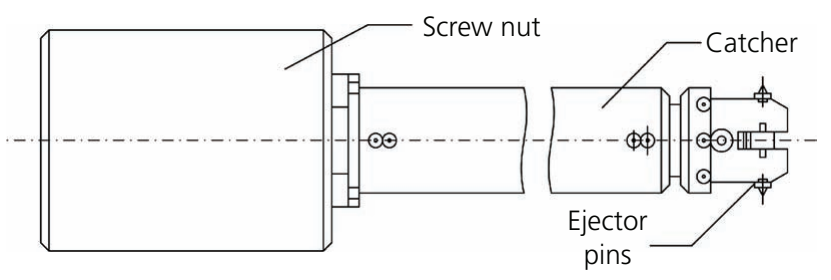

Figure 2. Structure of the catcher

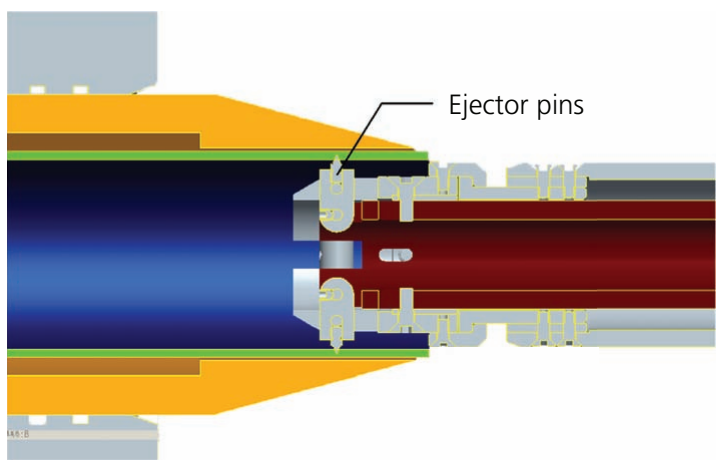

Figure 3. Working stage of the catcher

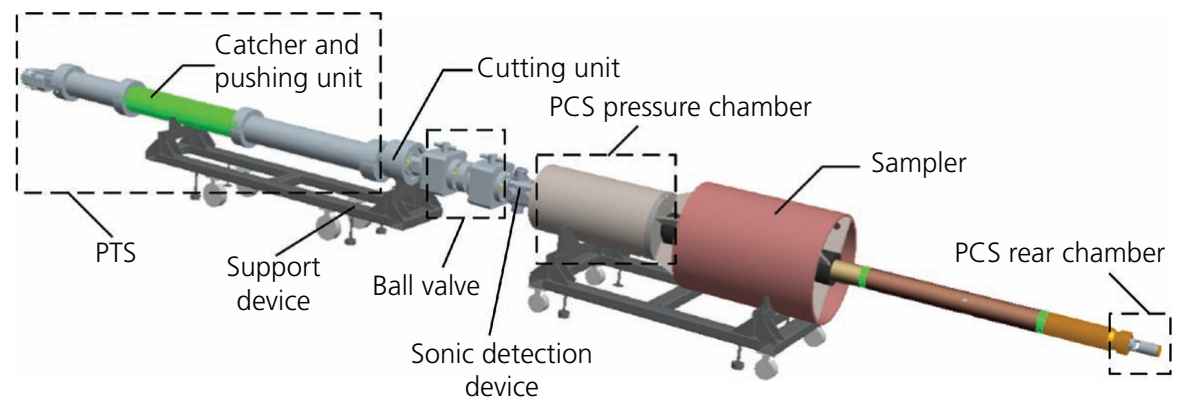

Figure 1. Components of the pressure-retained transfer device. PCS, pressure-retained coring system; PTS, pressure-retained transfer system 
Development of a pressure-retained transfer system of seafloor natural gas hydrates

Chen, Gao, Liu et al. reciprocating movement of the catcher. In order to facilitate the transportation and installation of the pressure-retained transfer device, the whole pressure-retained cylinder is divided into several segments and connected by half-structures.

The catcher mentioned earlier is fixed on the sliding nut. The sliding nut is connected with the screw through the inner thread and therefore moves in the axial direction only. The screw is limited by two end covers at the front and rear. The pushing unit is connected with the ball valve to realise the connection and separation of the pressure-retained transfer device and the corer or the sample canister.

\section{Cutting unit}

The cutting unit is composed of an oil-filled motor, a gear set, a core clamp and blades. The rotating motion of blades is driven by the motor, and the gripping motion of the clamp is realised by gripping ejectors, and the clamp is released by reset springs after gripping.

When cores are pulled into the cutting unit and reach a designated position, they can be gripped by the clamp, and then the feeding motion of blades is activated. The gripping ring is pushed by cutting ejectors, so cores can be cut round by blades. In this way, the impact to the bulk of cores is greatly reduced during the cutting process. The cutting unit is shown in Figure 4. In this paper, core liners are made of polycarbonate. Considering that most core liners are made of polycarbonate at present, the cutting unit has not been used to cut core liners of other special materials. After finishing the cutting motion, the cutting ejectors are dragged in the opposite direction, and the gripping ring is pushed back by the inner pressure and the reset springs.

\section{Subsampling unit}

The pressure-retained transfer system is used to cut and transfer cores obtained by the pressure-retained corer. However, in some experiments, smaller samples need to be extracted from the large cores. Therefore, a pressure-retained subsampling unit is required (Schultheiss et al., 2006). The configuration of the subsampling unit is shown in Figure 5. The hydraulic cylinder is used to

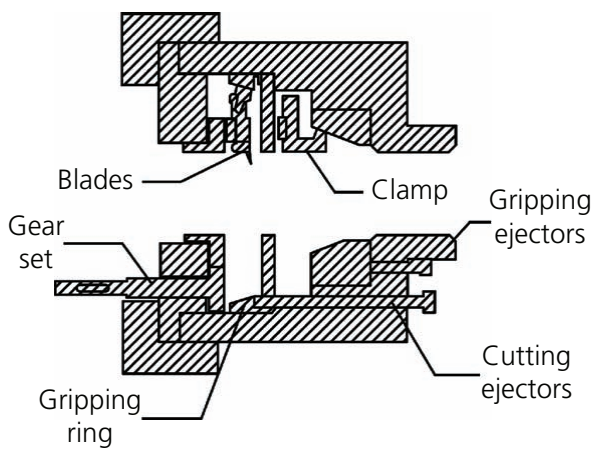

Figure 4. Structure of the cutting unit

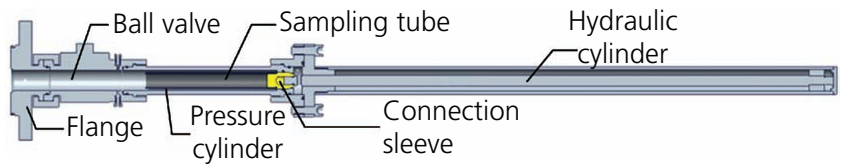

Figure 5. Structure of the subsampling unit

provide the impetus during subsampling. The pressure-retained cylinder is used to load the sample while maintaining the inner pressure. The hydraulic system includes an accumulator to compensate for the loss of the inner pressure. During the subsampling process, a flange is connected with the ball valve. The water is injected into the subsampling unit by a pump. Then, the hydraulic cylinder piston is pushed to the left side in Figure 5 by a manual pump. In this way, subsampling is accomplished.

\section{Pressure-retained unit}

The pressure-retained unit is designed to maintain the inner pressure during the transfer process. The fluctuation of the inner pressure is restricted to be less than $20 \%$. The design of the pressure-retained unit refers to a hydraulic system that has a hydraulic pump and an accumulator to maintain the inner pressure. In the system, the accumulator reduces the response time of the pressure fluctuation while the hydraulic pump is used to compensate for the leakage (Kong and Quan, 2004; Luo et al., 2011). The pressure gauges are used to display the pressure in the system in real time, and the pressure sensor is used to record the pressure changes in the system. The pressure-retained unit is shown in Figure 6. In this paper, the model of the high-pressure pump is WJ15.08-2.2-6, with a flow rate of $41 / \mathrm{min}$ and a maximum working pressure of 250 bar. The head of the pump is equipped with a pressure gauge to show the pressure in real time. In order to prevent corrosion by seawater, the material of the pump is 3161 . The manual pump is produced by the company Unipac Technology, the model is u-jb-3, and the maximum working pressure is 800 bar. The pressure sensor is a 522 series sensor manufactured by the company Huba Control, the model of which is $522.9 \mathrm{~K} 3 \mathrm{~S} 0$. The measuring range is $0-250$ bar, the output signal is $4-20 \mathrm{~mA}$, the working voltage is $7-33 \mathrm{~V}$ and the response time is less than $2 \mathrm{~ms}$. The model of the pressure gauge is Y-101A, with a measuring range $0-250$ bar.

After the core is transferred into the pressure-retained transfer device from the pressure-retained corer, ball valves 4 and 5 are closed, and, therefore, the corer is separated from the transfer device. Then, the pressure-retained cylinder is connected to the pressure-retained transfer device, in which the inner pressure is raised to the in situ pressure. After that, ball valve 5 is opened and the core is pushed into the pressure-retained cylinder. The manual pump pressures the tail of the corer, so that the inner core can be taken off from the corer by the pressure difference.

Vents 1 and 2 are used to discharge the air in the transfer device. Ball valve 1 is used to connect the tail of the corer with the 


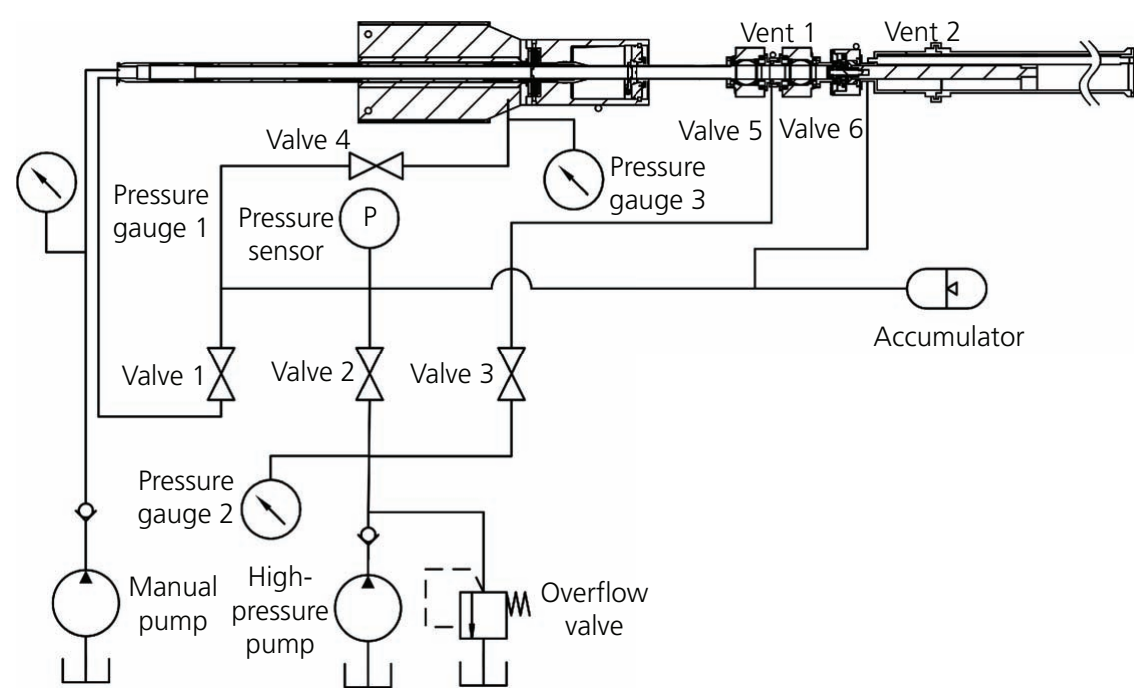

Figure 6. Diagram of the pressure-retained system

pressure-retained cylinder, so that the pressure fluctuation caused by the movement of core can be suppressed. Ball valve 2 controls the high-pressure pump and the transfer device. When ball valve 2 is opened, the transfer device is pumped with water and thus pressured. When ball valve 2 is closed, the pressure of transfer device is retained and isolated from the pressure fluctuations in the system. Water can be injected in the pressure-retained cylinder when ball valve 3 is open. When ball valve 3 is closed, the pressure in the system can be maintained. Ball valve 4 connects the corer and the pressure-retained system. Ball valves 5 and 6 connect the corer and the transfer device. The high-pressure pump provides power for the pressure-retained system and pumps water into the transfer device. When the pressure in the pipeline of the pressure-retained system exceeds the overflow pressure, the overflow valve opens to reduce the pressure. The bladder accumulator absorbs the pressure pulsation. The pressure gauges show the real-time pressure in the transfer device, and the pressure sensors measure the pressure curves in the transfer device for further analysis.

\section{Transfer process}

The working process of the pressure-retained transfer system is mainly divided into the following ten steps. In this process, the medium in the system is seawater, and enough seawater is pumped into the system to exhaust the air in the device. To ensure that the medium and core samples in the system are not contaminated, all the inlets and outlets of the transfer device are sealed to avoid the exchange of materials with the outside world. Figure 7 shows the working condition of the valve or the vent in each step. The circles indicate that the valve or the vent is open.

Step 1: ball valves 2 and 3 are opened, and then water is injected into the transfer device.

Step 2: vents 1 and 2 are closed after the pressure in the transfer device becomes equal to the pressure in the corer.

Step 3: ball valve 4 is opened to balance the pressure in the transfer device and the pressure in the corer chamber.

\begin{tabular}{|c|c|c|c|c|c|c|c|c|}
\hline Step & Valve 1 & Valve 2 & Valve 3 & Valve 4 & Valve 5 & Valve 6 & Vent 1 & Vent 2 \\
\hline 1 & & 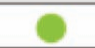 & 0 & & 0 & 0 & O & 0 \\
\hline 2 & & O & O & & O & O & & \\
\hline 3 & & 0 & 0 & O & 0 & 0 & & \\
\hline 4 & O & 0 & 0 & 0 & O & 0 & & \\
\hline 5 & O & 0 & O & O & O & 0 & & \\
\hline 6 & O & 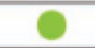 & & 0 & & & 0 & \\
\hline 7 & 0 & 0 & O & O & & & 0 & \\
\hline 8 & 0 & & O & 0 & & & & \\
\hline 9 & O & 0 & O & O & 0 & 0 & & \\
\hline 10 & 0 & O & & O & & & O & \\
\hline
\end{tabular}

Figure 7. Working state of valves and vents during the transfer process 
Development of a pressure-retained transfer system of seafloor natural gas hydrates

Chen, Gao, Liu et al.
Step 4: ball valve 1 is opened to balance the pressure in the transfer device and the pressure in the rear of corer.

Step 5: the core is transferred into the cutting unit and cut into segments.

Step 6: ball valves 3, 5 and 6 are closed, and vent 1 is opened; then, the corer is detached.

Step 7: the sample canister is connected to the transfer device, and then ball valve 3 is opened and water is injected into the canister.

Step 8: ball valve 2 and vent 1 are closed, and then the sample canister is pressured.

Step 9: ball valves 2, 5 and 6 are opened, and then the core is pushed into the sample canister by the catcher.

Step 10: ball valves 3, 5 and 6 are closed, and vent 1 is opened; then, the sample canister is detached.

\section{Performance of pressure retaining}

\section{Calculation of pressure fluctuation}

The movement of the catcher in the pressure-retained cylinder may cause pressure change, so it is necessary to calculate whether the pressure change causes pressure fluctuations in the system. A schematic diagram of the catcher movement is shown in Figure 8.

Using the flow rate equation for annular gap flow

$$
\text { 1. } Q=\frac{\pi d_{1} \delta^{3}}{12 \mu L} \Delta P=\frac{\pi d_{1} \delta^{3}}{12 \mu L}\left(P_{1}-P_{2}\right)
$$

When the catcher is propelled, the flow rate of the medium in the pressure-retained cylinder can be calculated as

2. $Q=\frac{\pi d_{0}^{2} v}{4}$

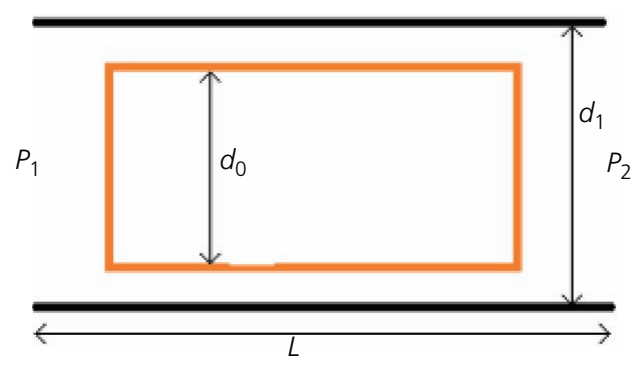

Figure 8. Diagram of the catcher movement where $P_{1}$ is the inlet pressure; $P_{2}$ is the outlet pressure; $d_{0}$ is the catcher diameter, which is $73 \mathrm{~mm} ; d_{1}$ is the hole diameter, which is $75 \mathrm{~mm} ; L$ is the catcher length, which is $1730 \mathrm{~mm} ; \mu$ is the dynamic viscosity of the medium, which is $999 \times 10^{-6} \mathrm{~Pa} \mathrm{~s}$; $v$ is the speed of catcher, which is $5.3 \mathrm{~mm} / \mathrm{s}$; and $\delta$ is the gap width, which is $10^{-3} \mathrm{~m}$. Thus, the flow rate of medium can be calculated as follows

$$
\begin{aligned}
Q & =\frac{3 \cdot 14 \times\left(73 \times 10^{-3}\right)^{2} \times 5 \cdot 3 \times 10^{-3}}{4} \\
\text { 3. } \quad & =2 \cdot 22 \times 10^{-5} \mathrm{~m}^{3} / \mathrm{s}
\end{aligned}
$$

The differential pressure can be calculated by using the flow rate equation for annular gap flow and the flow rate of the medium

$$
\begin{aligned}
\Delta P & =\frac{12 \times 999 \times 10^{-6} \times 1.73 \times 2.22 \times 10^{-5}}{3.14 \times 75 \times 10^{-3} \times 10^{-9}} \\
& =1.96 \mathrm{kPa}
\end{aligned}
$$

The pressure change of the screw nut can be calculated in the same way, yet noting that the physical meanings of parameters in Equations 1 and 2 have changed: $d_{0}$ is the diameter of the screw nut, which is $215 \mathrm{~mm} ; d_{1}$ is the hole diameter, which is $220 \mathrm{~mm}$; $L$ is the length of the screw nut, which is $90 \mathrm{~mm} ; \mu$ is the dynamic viscosity of the medium, which is $999 \times 10^{-6} \mathrm{~Pa} \mathrm{~s}$; $v$ is the speed of the screw nut, which is $5.3 \mathrm{~mm} / \mathrm{s}$; and $\delta$ is the gap width, which is $2.5 \times 10^{-3} \mathrm{~m}$.

According to Equation 1, the flow rate of the medium is

$$
\begin{aligned}
Q & =\frac{3 \cdot 14 \times\left(215 \times 10^{-3}\right)^{2} \times 5.3 \times 10^{-3}}{4} \\
5 . \quad & =1.9 \times 10^{-4} \mathrm{~m}^{3} / \mathrm{s}
\end{aligned}
$$

According to Equation 2, when the screw nut is propelled, the pressure difference is

$$
\begin{aligned}
\Delta P & =\frac{12 \times 999 \times 10^{-6} \times 0.09 \times 1.9 \times 10^{-4}}{3.14 \times 220 \times 10^{-3} \times\left(2.5 \times 10^{-3}\right)^{3}} \\
6 . \quad & =1.92 \mathrm{~Pa}
\end{aligned}
$$

It can be concluded from the above calculation that the pressure difference caused by the movement of catcher is larger than that caused by the movement of screw nut. The pressure difference can be neglected when compared with the inner pressure in the transfer system.

\section{Experiment on pressure retaining}

In order to verify the pressure-retaining performance of the transfer system during the whole transfer process, a laboratory pressure-retaining experiment is carried out. The inner pressure of the system is set to $21 \mathrm{MPa}$. 
Environmental Geotechnics

Volume 8 Issue 8
Development of a pressure-retained

transfer system of seafloor natural gas

hydrates

Chen, Gao, Liu et al.
In the first step of the transfer process, the core is separated from the corer. The separation procedure is carried out by pressuring the tail of the corer. The pressure change inside the transfer device during the docking process is shown in Figure 9. In the first 0-70 s, the transfer device is pressured by the high-pressure pump and the core is separated by the pressuring at the tail of the corer. At 70-2000 s, the core is pushed into the transfer device by the rear pressure, which causes a slow rise of the pressure in the transfer device. At 2000-2500 s, the catcher is moved towards the core, and this period is magnified in Figure 10. Because the gap between the catcher and the pressure-retained cylinder is small, the movement of the catcher causes the medium to flow. Therefore, a fluctuation of the pressure turns up, which has an amplitude of 16.5-22 MPa. The frequency of the fluctuation is much higher than that of pressure change during the transfer process. Because the pressure fluctuation is caused by the flow of medium next to the pressure sensors, this means that it is a kind of sensor disturbance. Moreover, it is less than $20 \%$ of the inner pressure. Thus, the pressure fluctuation can be ignored when the pressure change in the whole transfer process is focused on.

After the core is gripped, it is pulled into the cutting unit by the catcher. Then, the core is cut with the cutting part pushed into the sample canister. The curve of the pressure change in this process is shown in Figure 11. At $0-500 \mathrm{~s}$, the core is gripped by the catcher. At $500-1100 \mathrm{~s}$, the core is pulled to the cutting unit by the catcher. In this process, the origin of the pressure fluctuation is the same as the one in the catcher movement process mentioned earlier. At $600-800 \mathrm{~s}$, no pressure fluctuation is observed because the catcher arrives at the observation hole and stops moving. At $1400-1800 \mathrm{~s}$, the core is cut. This period is magnified in Figure 12. During the cutting process, the movement of blades causes the medium to flow, so the motion of cutting causes pressure fluctuation, the amplitude of which is $16 \cdot 8-23.7 \mathrm{MPa}$. The pressure change is less than $5 \%$ in the cutting process if the pressure fluctuation is ignored. This means that the performance of pressure maintenance is excellent. At $1800-2500 \mathrm{~s}$, the cutting part is pushed into the sample canister by the catcher. Similarly as in the preceding periods, the pressure fluctuates when the catcher moves, and the actual change in pressure is less than $5 \%$ after ignoring the fluctuation.

When the segment of the core is pushed into the sample canister after cutting, the core is detached from the catcher, and then the catcher is moved back to the transfer device. The pressure curve in this process is shown in Figure 13. At $0-100 \mathrm{~s}$, the core is pushed into the sample canister. At $100-500 \mathrm{~s}$, the core is separated from the catcher. At $500-800 \mathrm{~s}$, the catcher is pulled back into the transfer device. The pressure fluctuation is much smaller than the previous steps shown in Figure 10 because the sample canister is far from the pressure sensor. Because the pressure fluctuation is a kind of sensor disturbance, it is not the real change in inner pressure. When the distance between the sample and the pressure sensor becomes larger, the pressure

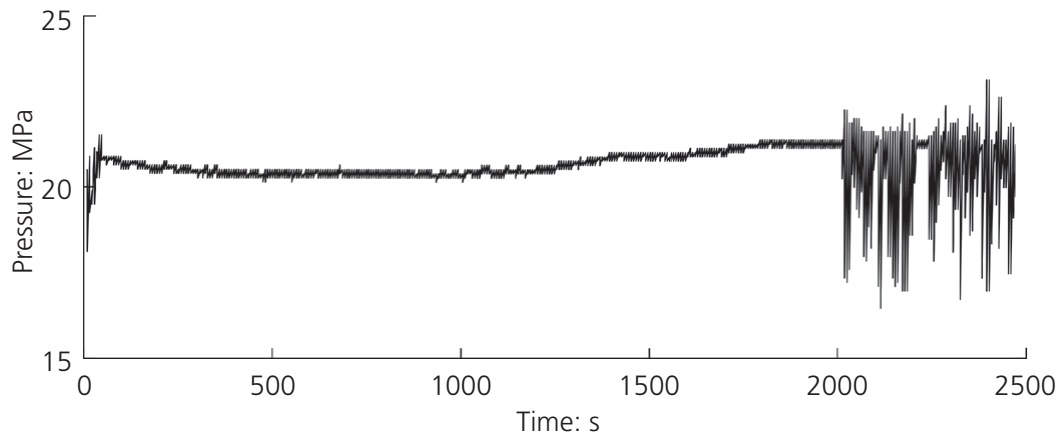

Figure 9. Pressure curve of the docking process

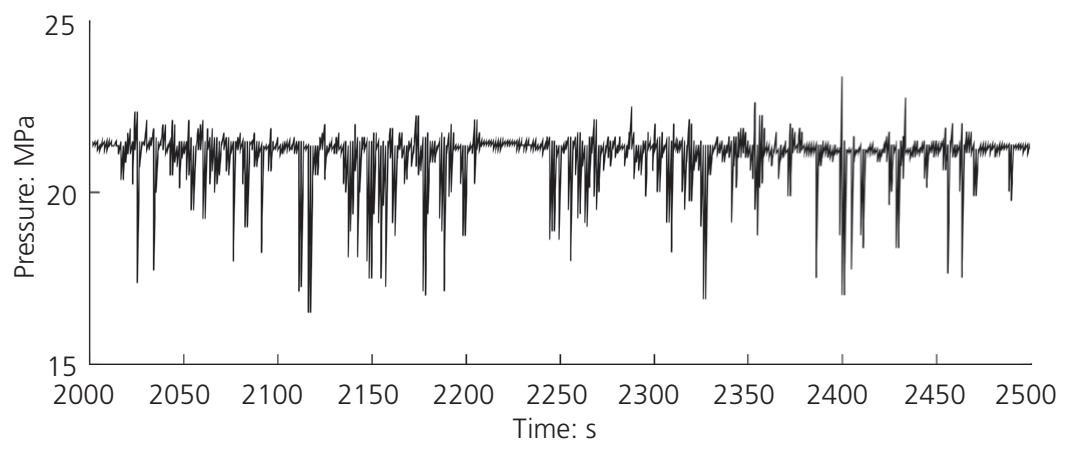

Figure 10. Pressure curve of the catcher movement 


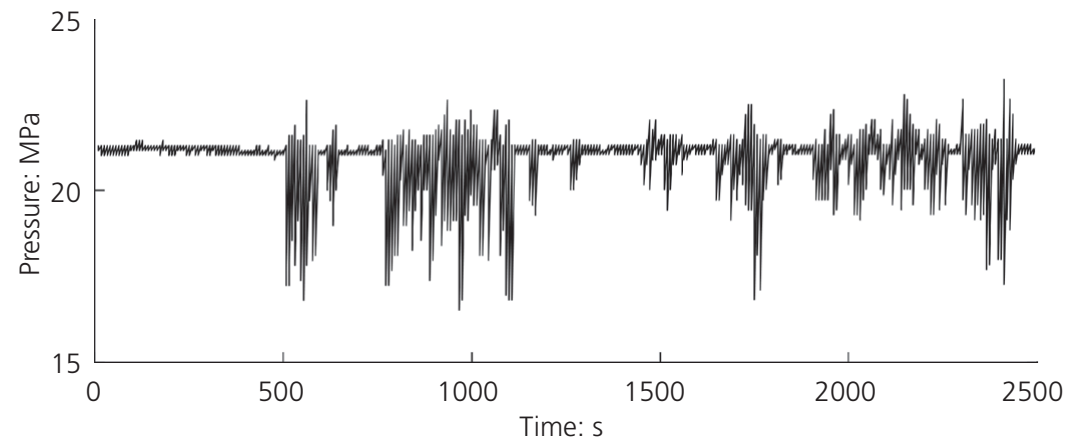

Figure 11. Pressure curve of the transfer process

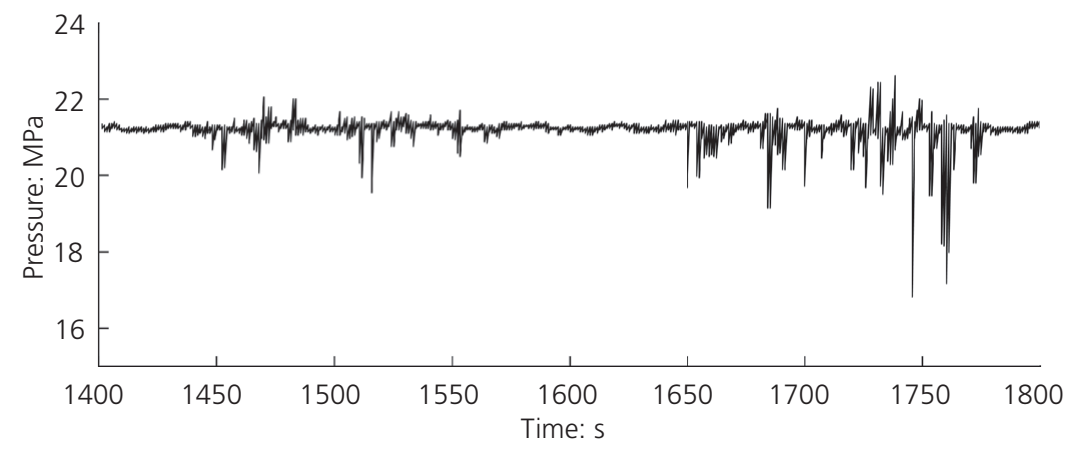

Figure 12. Pressure curve of the cutting process

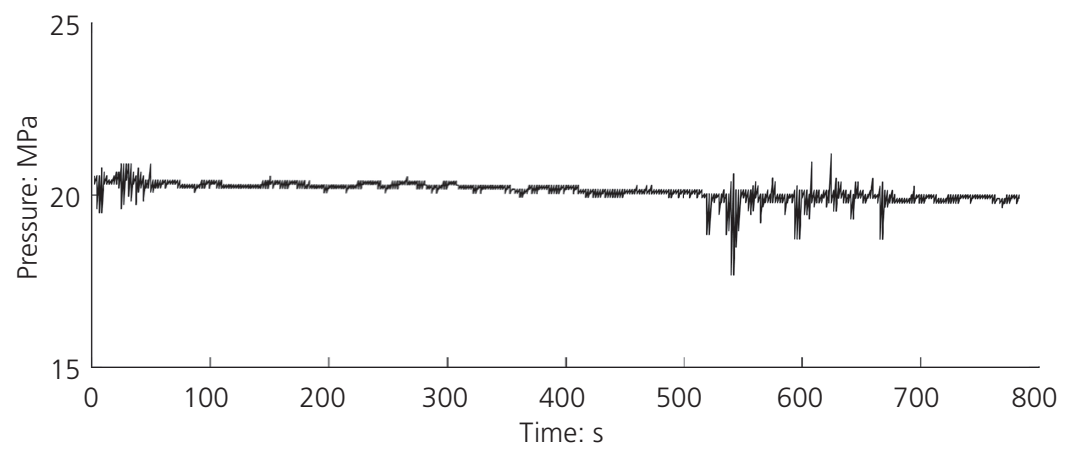

Figure 13. Pressure curve of the core separation process

fluctuation will be smaller, but it will not affect the inner pressure because the transfer process is a very slow process.

\section{Sea trials and discussion}

\section{Coring and transfer trials}

Two sites in the South China Sea are chosen for the sea trial of the authors' system. Both sites are located in a gas hydrate exploration area where the water depths are 2264 and $1130 \mathrm{~m}$ at site 1 and site 2, respectively.
The corer used in this sea trial is developed by Zhejiang University and Guangzhou Marine Geological Survey. The outer diameter of the core is $73 \mathrm{~mm}$, and the inner diameter is $67 \mathrm{~mm}$. The core has a length of $5215 \mathrm{~mm}$.

The first coring is taken at site 1 with a coring rate of $90 \%$ achieved. The inner pressure of the core is maintained at $4.5 \mathrm{MPa}$ initially. In order to adapt to the operating pressure of the transfer system, the pressure of the core is raised to $20 \mathrm{MPa}$ manually. The second coring is taken at site 2 . The coring rate rises to $99 \%$, and 
Environmental Geotechnics

Volume 8 Issue 8
Development of a pressure-retained

transfer system of seafloor natural gas

hydrates

Chen, Gao, Liu et al. the pressure of the core is maintained at $11 \mathrm{MPa}$. After coring, the transfer trial is carried out, as shown in Figure 14.

\section{Results}

In the transfer trial, four pressure sensors are set at the rear of the pressure corer, the pressure-retained transferring segment, the ball valve of transfer device and the pressure chamber of corer. The pressure change during the transfer process of the two transfer trials is measured by the pressure sensors. The pressure curves are shown in Figures 15 and 16.

Among the pressure curves, the pressure at the rear of the corer (red curve) is about $4 \mathrm{MPa}$ higher than the pressure in the transfer device (blue curve). This is because the pressure at the rear of corer is raised before the transfer trial, so that the core can be separated from the corer by the pressure difference. Except the initial pressurisation stage, the pressure at the ball valve (yellow curve) and the pressure in the corer chamber (green curve) are consistent with the pressure of the transfer device during the transfer process. The pressure fluctuation is caused by the movement of the catcher and cutting unit, the amplitude of which is less than $20 \%$. When the pressure at the ball valve drops to 0 , the process of transfer is finished, so the pressure rises immediately. In addition, it should be noted that in the first trial, the pressure in the transfer device is released when the transfer process is finished; therefore, the pressure drops down. In contrast, in the second trial, the pressure of the transfer device increases after the transfer process is finished since the high-pressure pumps are still working.

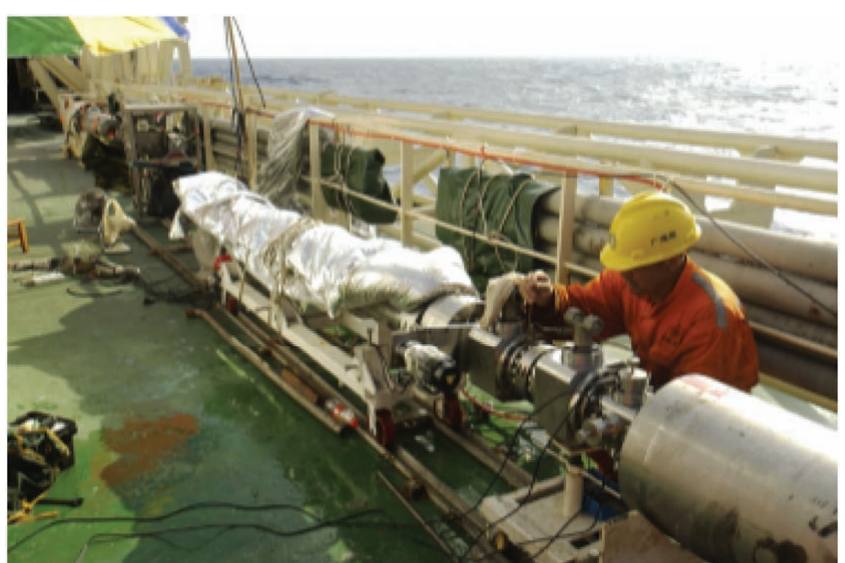

(a)

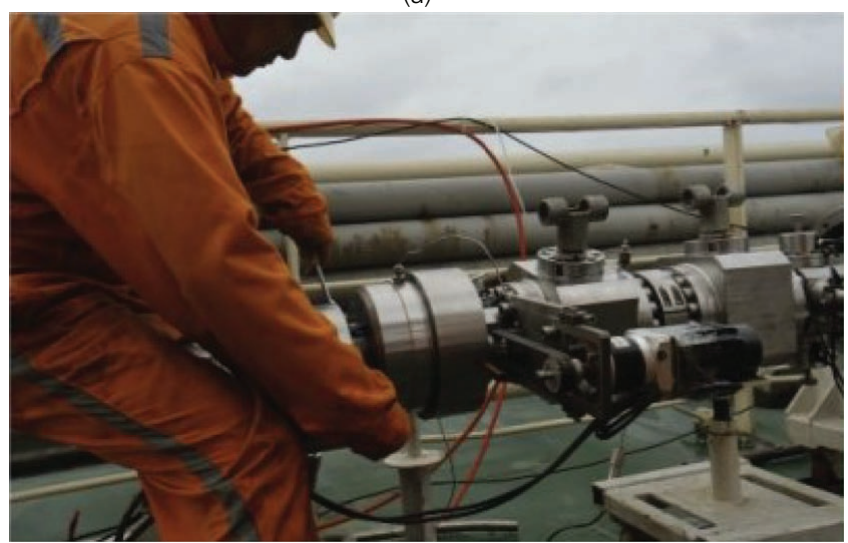

(c)

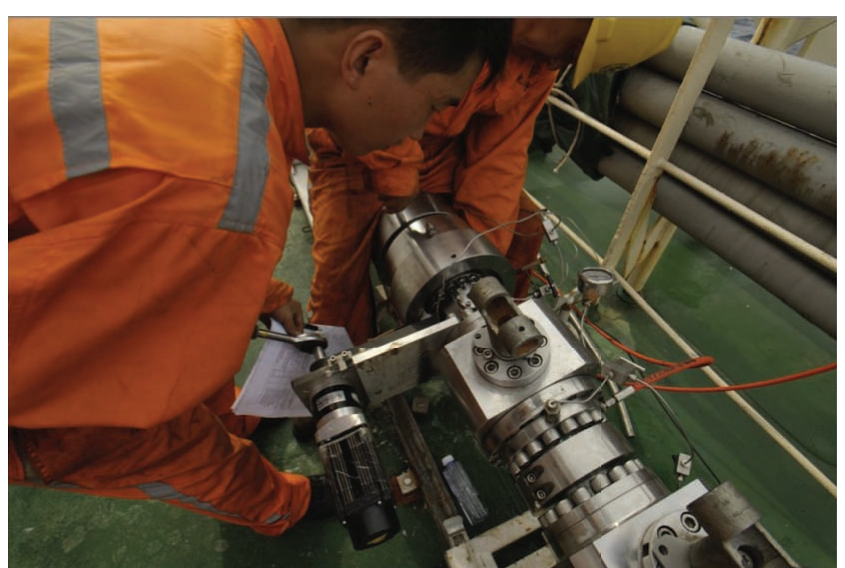

(b)

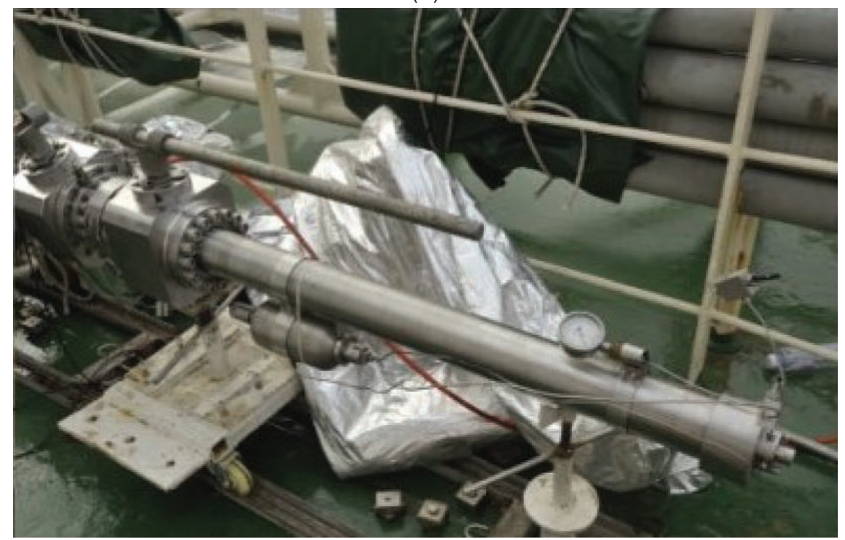

(d)

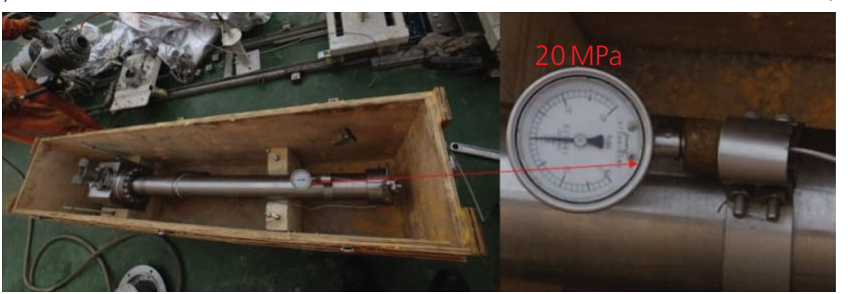

(e)

Figure 14. Photographs of the sea trial in 2016: (a) the corer is connected to the pressure-retained transfer system; (b) grabbing the core; (c) cutting the core; (d) the sample chamber is connected to the pressure-retained transfer system; (e) the transfer is complete 
Development of a pressure-retained transfer system of seafloor natural gas hydrates

Chen, Gao, Liu et al.

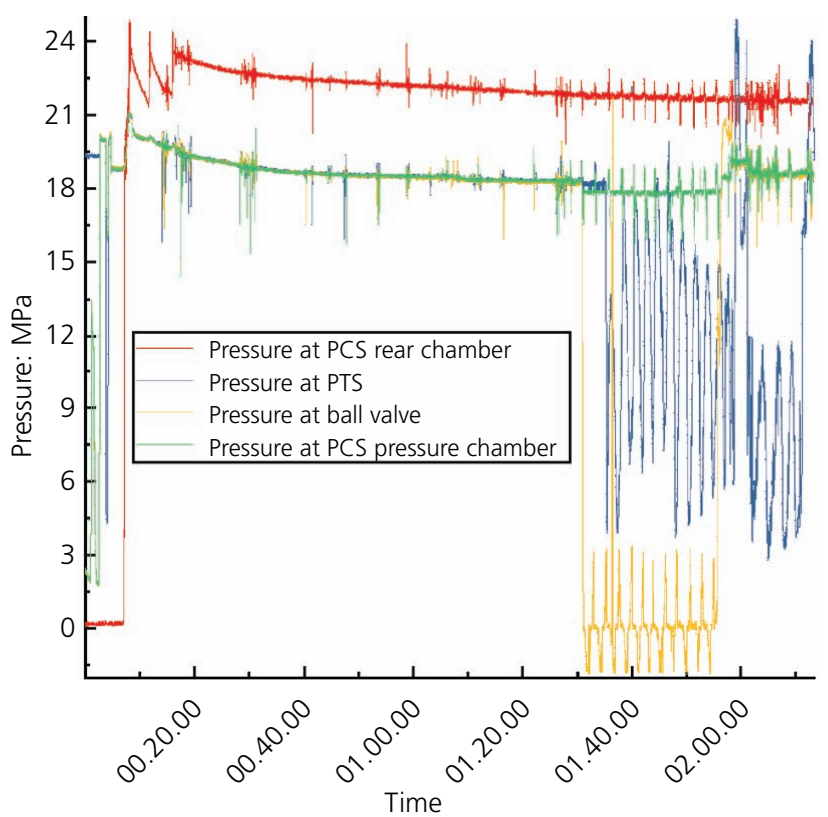

Figure 15. Pressure curves of the transfer process at site 1. PTS, pressure-retained transfer system

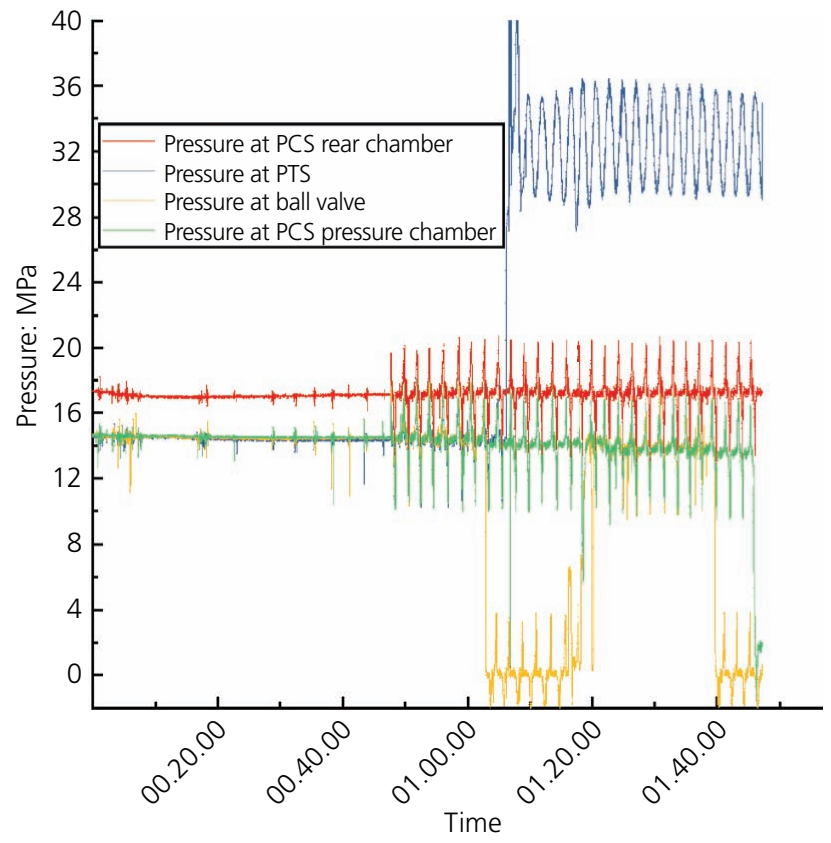

Figure 16. Pressure curves of the transfer process at site 2 . PTS, pressure-retained transfer system

\section{Outlook}

Long-columnar cores of deep-sea gas hydrates obtained by a corer can be transferred by the pressure-retained transfer system, which has the functions of core gripping, pushing, cutting and subsampling. During the transfer process, the inner pressure of the system is quite stable, regardless of the pressure fluctuation due to the transfer movement. Two pressure-retained coring and transfer trials have been carried out in the South China Sea. At the first trial, the pressure fluctuation in the transfer process was below $2 \mathrm{MPa}$ and was less than $1 \mathrm{MPa}$ in the second trial. The fluctuations are both less than $10 \%$ of the system pressure, which means that the transfer system has an excellent performance of pressure maintenance. Nevertheless, the pressure fluctuation caused by the movement of the catcher and cutting unit is still obvious and the temperature-retained performance of this system is still an issue, which are to be solved in further research.

\section{Acknowledgements}

This work was supported by the National Key Research and Development Program of China (number 2017YFC0307503) and the National High Technology Research and Development Program of China ('863; Program, number 2013AA092503). The authors greatly appreciate the support of the crews of the R/V Haiyang 6 in the sea trial.

\section{REFERENCES}

Abegg F, Hohnberg HJ, Pape T, Bohrmann G and Freitag J (2008) Development and application of pressure-core-sampling systems for the investigation of gas- and gas-hydrate-bearing sediments. Deep-sea Research, Part I Oceanographic Research Papers 55(11): 1590-1599, https://doi.org/10.1016/j.dsr.2008.06.006.

Chen JW, Fan W, Bingham B et al. (2013) A long gravity-piston corer developed for seafloor gas hydrate coring utilizing an in situ pressureretained method. Energies 6(7): 3353-3372, https://doi.org/10.3390/ en6073353.

Hao ZB, Huang WQ, Qin JX and Wu XY (2013) Estimation technique for gas hydrate saturation of pressure core samples. Chinese Journal of Geophysics - Chinese Edition 56(11): 3917-3921, https://doi.org/10. 6038/cjg20131133.

Jutzeler M, White JDL, Talling PJ et al. (2014) Coring disturbances in IODP piston cores with implications for offshore record of volcanic events and the Missoula megafloods. Geochemistry, Geophysics, Geosystems 15(9): 3572-3590, https://doi.org/10.1002/ $2014 \mathrm{GC} 005447$.

Kong X and Quan L (2004) The history, current situation of accumulator's research and its vista. Machine Tool \& Hydraulics 2004(10): 4-6.

Luo X, Shi Z, Hu J and Niu Z (2011) Semi-active accumulator absorbing pressure pulsation in high-pressure water-jet propulsion system. Proceedings of the Institution of Mechanical Engineers, Part C: Journal of Mechanical Engineering Science 225(9): 2052-2061, https://doi.org/10.1177\%2F0954406211405925.

Priest JA, Druce M, Roberts J et al. (2015) PCATS triaxial: a new geotechnical apparatus for characterizing pressure cores from the Nankai Trough, Japan. Marine and Petroleum Geology 66: 460-470, https://doi.org/10.1016/j.marpetgeo.2014.12.005.

Rothwell RG and Rack FR (2006) New techniques in sediment core analysis: an introduction. Geological Society, London, Special Publications 267(1): 1-29, https://doi.org/10.1144/GSL.SP.2006.267. 01.01.

Santamarina JC, Dai S, Jang J and Terzariol M (2012) Pressure core characterization tools for hydrate-bearing sediments. Scientific Drilling 16(4): 44-48, https://doi.org/10.2204/iodp.sd.14.06.2012.

Santamarina JC, Dai S, Terzariol M et al. (2015) Hydro-bio-geomechanical properties of hydrate-bearing sediments from Nankai Trough. Marine and Petroleum Geology 66(2): 434-450, https://doi.org/10.1016/j. marpetgeo.2015.02.033. 
Environmental Geotechnics

Volume 8 Issue 8
Development of a pressure-retained

transfer system of seafloor natural gas

hydrates

Chen, Gao, Liu et al.
Schultheiss PJ, Francis TJG, Holland M et al. (2006) Pressure coring, logging and subsampling with the hyacinth system. Geological Society, London, Special Publications 267(1): 151-163, https://doi.org/ 10.1144/GSL.SP.2006.267.01.11.

Schultheiss P, Holland M and Humphrey G (2009) Wireline coring and analysis under pressure: recent use and future developments of the HYACINTH system. Scientific Drilling 3(7): 44-50, https://doi.org/10. 5194/sd-7-44-2009.

Tréhu A, Ruppel C, Holland M et al. (2006) Gas hydrates in marine sediments: lessons from scientific ocean drilling. Oceanography 19(4) 124-142, https://doi.org/10.5670/oceanog.2006.11.

Yamamoto K (2015) Overview and introduction: pressure core-sampling and analyses in the 2012-2013 MH21 offshore test of gas production from methane hydrates in the eastern Nankai Trough. Marine and Petroleum Geology 66(Part 2): 296-309, https://doi.org/10.1016/j. marpetgeo.2015.02.024.
Yoneda J, Masui A, Tenma N and Nagao J (2013) Triaxial testing system for pressure core analysis using image processing technique. Review of Scientific Instruments 84(11): 114503, https://doi.org/10.1063/1. 4831799.

Yoneda J, Masui A, Konno Y et al. (2017) Pressure-core-based reservoir characterization for geomechanics: insights from gas hydrate drilling during 2012-2013 at the eastern Nankai Trough. Marine and Petroleum Geology 86(2017): 1-16, https://doi.org/10.1016/j. marpetgeo.2017.05.024

Yun TS, Lee C, Lee JS, Bahk JJ and Santamarina JC (2011) A pressure core based characterization of hydrate-bearing sediments in the Ulleung Basin, Sea of Japan (East Sea). Journal of Geophysical Research 116(B2): article B02204, https://doi.org/10.1029/2010JB007468.

Zhu H, Liu Q, Deng J et al. (2011) Pressure and temperature preservation techniques for gas-hydrate-bearing sediments sampling. Energy 36(7): 4542-4551, https://doi.org/10.1016/j.energy.2011.03.053.

\section{How can you contribute?}

To discuss this paper, please submit up to 500 words to the editor at journals@ice.org.uk. Your contribution will be forwarded to the author(s) for a reply and, if considered appropriate by the editorial board, it will be published as a discussion in a future issue of the journal. 